\title{
Networked Convergence of Fractional-Order Multiagent Systems with a Leader and Delay
}

\author{
Yuntao Shi ${ }^{1}$ and Junjun Zhang ${ }^{2}$ \\ ${ }^{1}$ Key Laboratory of Beijing for Field-Bus Technology \& Automation, North China University of Technology, Beijing 100144, China \\ ${ }^{2}$ College of Science, North China University of Technology, Beijing 100144, China \\ Correspondence should be addressed to Yuntao Shi; shiyuntao@ncut.edu.cn
}

Received 27 August 2015; Accepted 5 October 2015

Academic Editor: Michael Z. Q. Chen

Copyright (c) 2015 Y. Shi and J. Zhang. This is an open access article distributed under the Creative Commons Attribution License, which permits unrestricted use, distribution, and reproduction in any medium, provided the original work is properly cited.

\begin{abstract}
This paper investigates the convergence of fractional-order discrete-time multiagent systems with a leader and sampling delay by using Hermite-Biehler theorem and the change of bilinearity. It is shown that such system can achieve convergence depending on the sampling interval $h$, the fractional-order $\alpha$, and the sampling delay $\tau$ and its interconnection topology. Finally, some numerical simulations are given to illustrate the results.
\end{abstract}

\section{Introduction}

Recently, more and more scholars focus on the coordinated control $[1,2]$ of multiagent systems such as the consensus [3$5]$ and the controllability [6-8]. However, most of the practical distribution systems are fractional order [9-12]. Recently, with the development of society, fractional-order calculus theory [13-16] is widely used to study the signal processing and control, picture processing and artificial intelligence, and so on. The consensus of multiagent systems refers to the fact that agents in the system can transfer information and influence each other according to a certain protocol or algorithm, and eventually agents will tend to the consensus behavior with the evolution of the time in [17]. In fact, for most of multiagent systems, there widely exist time delays as in [18]. So the property of multiagent systems with time delays has always been the hot problem. In [19], the authors studied consensus of multiagent systems with heterogeneous delays and leader-following with integer-order and continuous time. In [20], the paper considered the consensus of fractionalorder multiagent systems with sampling delays without the leader.

However, for a complex environment, multiagent systems with fractional-order can be better to describe some real natural phenomena. Some basic issues of fractional-order multiagent systems with time delay, such as the convergence, are still lacking in studying. Specially, for a fractional-order multiagent system, which depends crucially on sampling interval $h$, the fractional-order $\alpha$, and its interconnection topology, therefore, it is more difficult to study the convergence of the fractional-order multiagent system.

In this paper, we consider the convergence of fractionalorder discrete-time multiagent systems with a leader and sampling delay. The leader plays the role of an external input or signal to followers, and the followers update their states based on the information available from their neighbors and the leader. We will establish convergence conditions and discuss relations among sampling interval $h$, the fractionalorder $\alpha$, its sampling delay $\tau$, and its interconnection topology of such network.

The remainder of this paper is organized as follows. Section 2 gives the model and some preliminaries. Section 3 presents the main results, and some simulations are given in Section 4. Finally, Section 5 gives the conclusion.

\section{Preliminaries and Problem Statement}

In this section, we introduce some useful concepts and notations about the definition of fractional derivative [21], graph theory, and convergence of the multiagent systems. 
Denote a directed graph as $\mathscr{G}=(\mathscr{V}, \mathscr{E}, A)$ consisting of a nonempty set of vertices $\mathscr{V}$ and $\mathscr{E}=\{(i, j): i, j \in \mathscr{V}\}$ is a set of edges, where $(i, j)$ means an arc starts from $i$ and ends by $j$. If $i, j \in \mathscr{V}$ and $(i, j) \in \mathscr{E}$, then we say that $i$ and $j$ are adjacent or $j$ is a neighbor of $i$. We make $\mathcal{N}_{i}=\{i \in \mathscr{V}:(i, j) \in \mathscr{E}\}$ be the neighborhood set of node $i . A=\left[a_{i j}\right]$ is an adjacency matrix of graph $\mathscr{G}$, where $a_{i j} \geq 0$ is the coupling weight between any two agents. $D=\operatorname{diag}\left\{d_{1}, d_{2}, \ldots, d_{n}\right\} \in \mathbb{R}^{n \times n}$ is a degree matrix of $\mathscr{G}$; its diagonal elements $d_{i}=\sum_{j \in \mathcal{N}_{i}} a_{i j}, i=$ $1,2, \ldots, n$, for the graph. Then the Laplacian of the weighted graph $\mathscr{G}$ is defined as

$$
L=D-A \in \mathbb{R}^{n \times n}
$$

The agent $i$ is a globally reachable agent if it has paths to all of other agents.

Definition 1 (see [17]). Assume that, for arbitrary given initial values, if

$$
\lim _{t \rightarrow \infty}\left(x_{i}(t)-s_{i} x_{0}(t)\right)=0
$$

$i \in \mathbb{N}$, where $x_{i}(t) \in \mathbb{R}^{n}$ is the state value of agent of the multiagent system $i(i \in \mathbb{N}, \mathbb{N}$ presents an index set
$(1,2, \ldots, N)), x_{0}(t) \in \mathbb{R}^{n}$, and $s_{i}$ is a constant which is changed with different $i$. Then we have that the multiagent system is convergence.

Definition 2 (see [21] (Grunwald-Letnikov)). For any real number $\alpha$, the integer part written for $\alpha$ is $[\alpha]$. If the function $f(t)$ has continuous $(m+1)$-order derivative in the interval $[\alpha, t]$ and $m$ equals $[\alpha]$ at last when $\alpha>0$, then let $\alpha$-order derivative be

$$
f^{(\alpha)}(t)=\lim _{h \rightarrow 0} h^{-\alpha} \sum_{i=0}^{(t-\alpha) / h}(-1)^{i}\left(\begin{array}{c}
-\alpha \\
i
\end{array}\right) f(t-i h) .
$$

Consider a multiagent system is composed of $N+1$ agents, where the first $N$ (labeled from 1 to $N$ ) are followers and the remainder agent $N+1$ (labeled 0 ) is leader. The fractionalorder discrete-time multiagent system with a leader and sampling time is described by

$$
\begin{aligned}
& x_{i}(k+1)=\alpha x_{i}(k)+h^{\alpha} u_{i}(k), \\
& x_{0}(k+1)=x_{0}(k),
\end{aligned}
$$

where

$$
u_{i}(k)= \begin{cases}\sum_{j \in \mathcal{N}_{i}} a_{i j}\left(x_{j}(k-1)-x_{i}(k-1)\right)+b_{i 0}\left(x_{0}(k-1)-x_{i}(k-1)\right), & t \in[k h, k h+\tau), \\ \sum_{j \in \mathcal{N}_{i}} a_{i j}\left(x_{j}(k)-x_{i}(k)\right)+b_{i 0}\left(x_{0}(k)-x_{i}(k)\right), & t \in[k h+\tau(k+1) h+\tau),\end{cases}
$$

$\alpha \in(0,1), x_{i} \in \mathbb{R}^{n}$ is the state of follower $i(i \in \mathbb{N}, \mathbb{N}$ presents an index set $(1,2, \ldots, N))$, and $x_{0} \in \mathbb{R}^{n}$ is the state of the leader. $\mathcal{N}_{i}$ is the neighbor set of agent $i . a_{i j} \geq 0$, $b_{i 0} \geq 0$ represent the coupling information between followers and from the leader to the followers, respectively; otherwise, $a_{i j}=0$ and $b_{i 0}=0 ; h>0$ is the sampling interval and the sampling interval is $h$ and the sampling delay is $0<\tau<h$.

\section{Main Results}

Let $X(k)=\left(x_{1}(k), x_{2}(k), \ldots, x_{N}(k)\right)^{T}$ and $X_{0}(k)=X_{0}(k+$ $1)$ be the state vectors of all the followers and the leader, respectively. Then such system can be rewritten as

$$
\left(\begin{array}{c}
X(k+1) \\
X(k)
\end{array}\right)=U\left(\begin{array}{c}
X(k) \\
X(k-1)
\end{array}\right)+\left(\begin{array}{c}
h^{\alpha} B \mathbf{1} X_{0}(k) \\
0
\end{array}\right)
$$

where

$$
U=\left(\begin{array}{cc}
\alpha I_{n}-h^{\alpha}(1-\tau)(L+B) & -h^{\alpha} \tau(L+B) \\
I_{n} & 0
\end{array}\right)
$$

$I_{N}$ is the $N \times N$ identity matrix, $B=\operatorname{diag}\left\{b_{10}, b_{20}, \ldots, b_{N 0}\right\} \in$ $\mathbb{R}^{N \times N}, \mathbf{1}=(1,1, \ldots, 1)^{T}$ is the $N \times 1$ identity matrix, and $L=\left[l_{i j}\right] \in \mathbb{R}^{N \times N}$ is Laplacian matrix with

$$
l_{i j}= \begin{cases}-a_{i j}, & i \neq j, \quad j \in \mathcal{N}_{i}, \\ \sum_{j \in \mathcal{N}_{i}} a_{i j}, & i=j, \\ 0, & \text { otherwise. }\end{cases}
$$

Lemma 3 ((Hermite-Biehler theorem) [22]). Assume the polynomial $q(s)=\rho_{0}+\rho_{1} s+\cdots+\rho_{n} s^{n}$, marking $q(j \omega)=$ $m(\omega)+j n(\omega)$. So $q(s)$ is Hurwitz stable if and only if the roots of $m(\omega)=0, m_{1}<m_{2}<\cdots$, and $n(\omega)=0, n_{1}<n_{2}<\cdots$ satisfy

$$
\begin{aligned}
& \text { (1) } m(0) n^{\prime}(0)-m^{\prime}(0) n(0)>0 \\
& \text { (2) } m_{1}<n_{1}<m_{2}<n_{2}<\cdots \text { or } n_{1}<m_{1}<n_{2}<m_{2}< \\
& \quad \cdots \text {. }
\end{aligned}
$$

Lemma 4 (see [23]). $\|M\|_{2}=\rho(M)$, if $M \in \mathbb{R}^{N \times N}$ is a symmetrical matrix.

Theorem 5. Suppose system (4) is a symmetrical and directly weighted network and the leader is a globally reachable agent; 


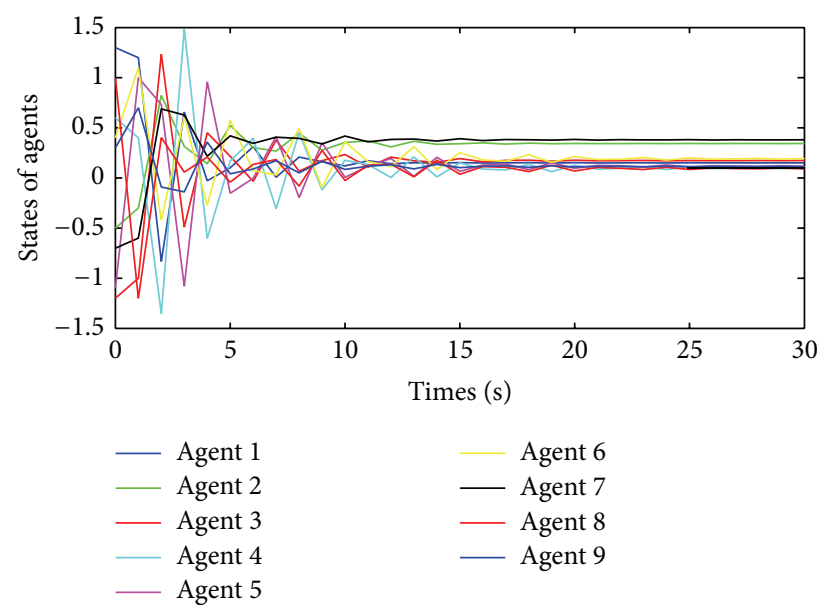

FIGURE 1: The trajectories of nine agents in the dynamical network.

then the state of each agent can converge to the spanning space of the leader's state, if and only if $\tau<\min \{1 / 2, h\}$ and

$$
h<\min \left\{\left(\frac{1+\alpha}{\lambda_{n}(1-2 \tau)}\right)^{1 / \alpha}, \frac{1}{\sqrt[\alpha]{\lambda_{n} \tau}}\right\}
$$

where $\lambda_{n}$ is the biggest eigenvalue of matrix $(L+B)$.

Proof. The fractional-order multiagent systems with sampling delay can be convergence if and only if $\|U\|<1$ which means the eigenvalues of matrix $U$ are less than 1 . Because the symmetrically directed weighted network at least has a globally reachable agent, $(L+B)$ can be orthogonal similar to a diagonal matrix. There exists an orthogonal matrix $P$ which makes

$$
L+B=P \Lambda P^{-1}
$$

where $\Lambda=\operatorname{diag}\left\{\lambda_{1}, \lambda_{2}, \ldots, \lambda_{n}\right\}$ and the eigenvalues of matrix $(L+B)$ satisfy $0<\lambda_{1} \leq \lambda_{2} \leq \cdots \leq \lambda_{n}$. Assuming that $z$ is the eigenvalue of matrix $U$, then the characteristic polynomial of $U$ is

$$
\begin{aligned}
& \operatorname{det}\left(z I_{2 n \times 2 n}-U\right) \\
& =\operatorname{det}\left(\begin{array}{cc}
z I_{n}-\alpha I_{n}+h^{\alpha}(1-\tau)(L+B) & h^{\alpha} \tau(L+B) \\
-I_{n} & z I_{n}
\end{array}\right) \\
& =\operatorname{det}\left(z^{2} I_{n}-z\left(\alpha I_{n}-h^{\alpha}(1-\tau)(L+B)\right)\right. \\
& \left.+h^{\alpha} \tau(L+B)\right) \\
& =\operatorname{det}\left(z^{2} I_{n}-z \alpha I_{n}+\left(z h^{\alpha}(1-\tau)+h^{\alpha} \tau\right)(L+B)\right) \\
& =\prod_{i \in N}\left(z^{2}-z \alpha+\left(z h^{\alpha}(1-\tau)+h^{\alpha} \tau\right) \lambda_{i}\right) \\
& \triangleq a(z) \text {. }
\end{aligned}
$$

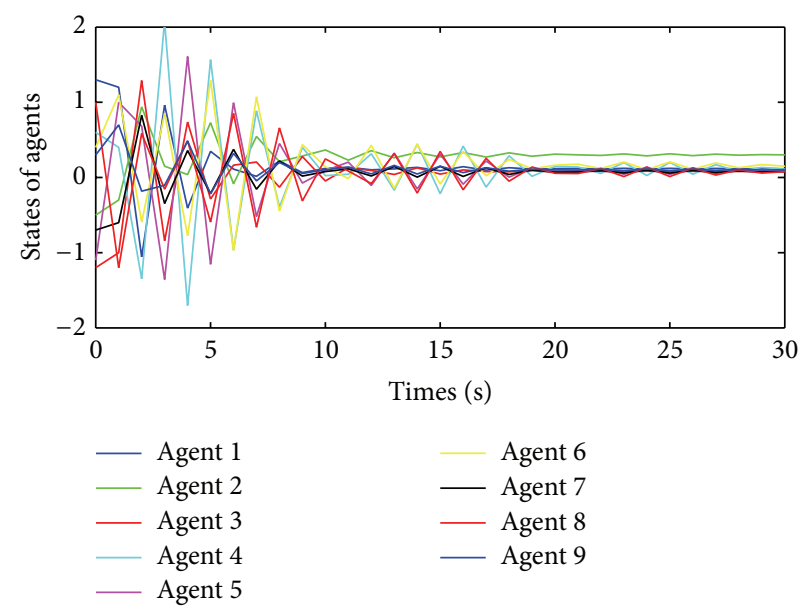

FIGURE 2: The trajectories of nine agents in the dynamical network.

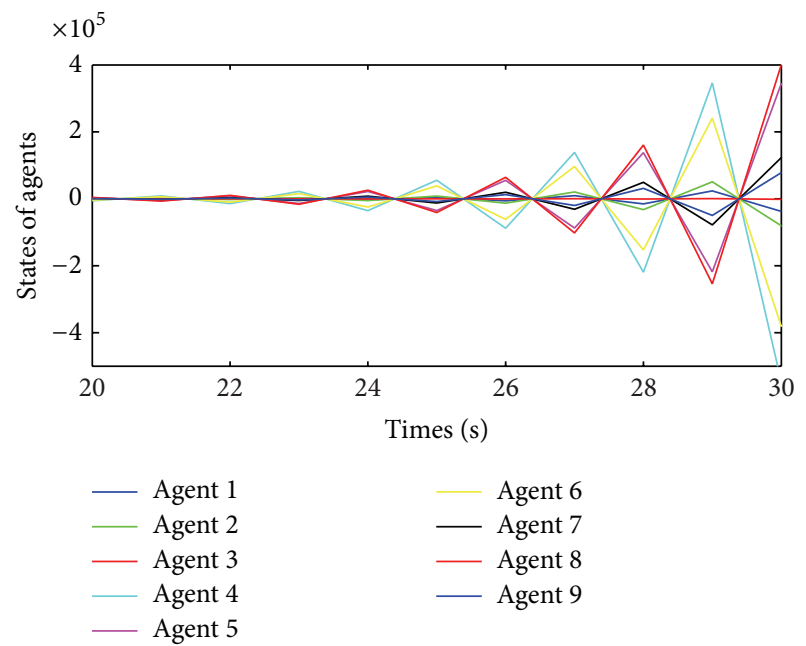

FIGURE 3: The trajectories of nine agents in the dynamical network.

Let $a(z)=0$; applying the double linear change $z=(s+$ $1) /(s-1)$, we can have

$$
\begin{aligned}
b(s)= & \left(1-\alpha+h^{\alpha} \lambda_{i}\right) s^{2}+\left(2-2 h^{\alpha} \tau \lambda_{i}\right) s+1+\alpha \\
& -h^{\alpha} \lambda_{i}+2 h^{\alpha} \tau \lambda_{i} .
\end{aligned}
$$

Since the network is symmetrical and directed, $(L+B)$ can be orthogonally similar to a diagonal matrix, whose eigenvalues are all positive. Let $s=j \omega$; then

$$
\begin{aligned}
b(j \omega)= & -\left(1-\alpha+h^{\alpha} \lambda_{i}\right) \omega^{2}+j\left(2-2 h^{\alpha} \tau \lambda_{i}\right) \omega+1 \\
& +\alpha-h^{\alpha} \lambda_{i}+2 h^{\alpha} \tau \lambda_{i} .
\end{aligned}
$$

Denote $b(\omega)=m(\omega)+j n(\omega)$, where

$$
\begin{aligned}
& m(\omega)=-\left(1-\alpha+h^{\alpha} \lambda_{i}\right) \omega^{2}+1+\alpha-h^{\alpha} \lambda_{i}+2 h^{\alpha} \tau \lambda_{i}, \\
& n(\omega)=\left(2-2 h^{\alpha} \tau \lambda_{i}\right) \omega .
\end{aligned}
$$




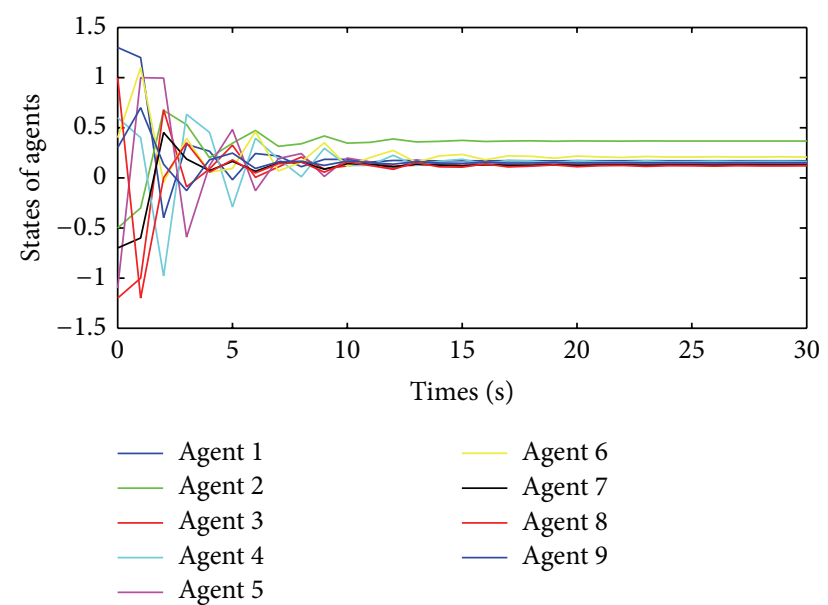

FIgURE 4: The trajectories of nine agents in the dynamical network.

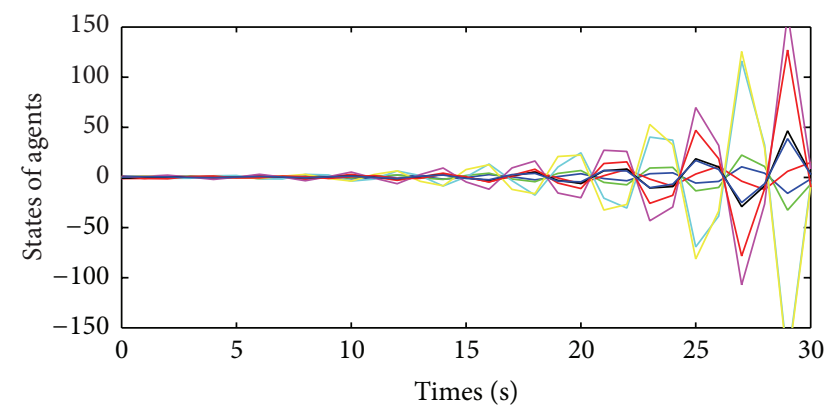

$\begin{array}{rr}- \text { Agent 1 } & \text { Agent 6 } \\ - \text { Agent 2 } & \text { Agent 7 } \\ \text { Agent 3 } & - \text { Agent 8 } \\ \text { Agent 4 } & - \text { Agent 9 } \\ - \text { Agent 5 } & \end{array}$

FIGURE 5: The trajectories of nine agents in the dynamical network.

Using Lemma 3, system (4) can be asymptotic convergence if and only if we have the following:

(1) if $m(0) n^{\prime}(0)-m^{\prime}(0) n(0)>0$, then $1+\alpha-h^{\alpha} \lambda_{i}+$ $2 h^{\alpha} \tau \lambda_{i}>0,1-h^{\alpha} \tau \lambda_{i}>0$, and then

$$
h^{\alpha}<\frac{1+\alpha}{\lambda_{i}(1-2 \tau)}, \quad h^{\alpha}<\frac{1}{\left(\tau \lambda_{i}\right)}
$$

(2) if $1-h^{\alpha} \tau \lambda_{i} \neq 0$, then the roots of $n(\omega)$ and $m(\omega)$ are the same, where the roots of $m(\omega)$ satisfy

$$
m^{2}(\omega)=\frac{\left(1+\alpha-h^{\alpha} \lambda_{i}+2 h^{\alpha} \tau \lambda_{i}\right)}{\left(1-\alpha+h^{\alpha} \lambda_{i}\right)}
$$

with $1+\alpha-h^{\alpha} \lambda_{i}+2 h^{\alpha} \tau \lambda_{i}>0$. Therefore, system (4) is asymptotic convergence.

Theorem 6. Suppose system (4) is a symmetrical and directly weighted network and the leader is a globally reachable agent; then the state of each agent can converge to the spanning space of the leader's state, if $\rho(A)<1$, where $A=h^{\alpha} \tau(L+B)$ and $\rho(A)$ is spectral radius of matrix $A$.
Proof. The fractional-order multiagent systems with sampling delay can be convergence if and only if $\|U\|<1$. Using Lemma 4,

$$
\begin{aligned}
& \|U\| \\
& =\left\|\left(\begin{array}{cc}
\alpha I_{n}-h^{\alpha}(1-\tau)(L+B) & I_{n} \\
I_{n} & 0
\end{array}\right)\left(\begin{array}{cc}
I_{n} & 0 \\
0 & -h^{\alpha} \tau(L+B)
\end{array}\right)\right\| \\
& \leq\left\|\left(\begin{array}{cc}
\alpha I_{n}-h^{\alpha}(1-\tau)(L+B) & I_{n} \\
I_{n} & 0
\end{array}\right)\right\|\left\|\left(\begin{array}{cc}
I_{n} & 0 \\
0 & -h^{\alpha} \tau(L+B)
\end{array}\right)\right\| \\
& =\rho\left(\begin{array}{cc}
\alpha I_{n}-h^{\alpha}(1-\tau)(L+B) & I_{n} \\
I_{n} & 0
\end{array}\right) \rho\left(\begin{array}{cc}
I_{n} & 0 \\
0 & -h^{\alpha} \tau(L+B)
\end{array}\right) \\
&
\end{aligned}
$$

Remark 7. Notice from Theorem 6 that $\|\cdot\|$ is $\|\cdot\|_{2}$ and $\|\cdot\|_{2}$ is 2 -norm.

Remark 8. Theorem 5 describes the relation of the convergence of such system and time delay, while Theorem 6 describes the relation of the convergence of such system and spectral radius of matrix $A$.

\section{Simulations}

In this section, we will present numerical simulations to illustrate the theoretical results.

Consider a multiagent system with nine agents and a leader, in which agent 0 is the leader and the rest are followers. The coupling matrix is defined as follows:

$$
\begin{aligned}
A & =\left[\begin{array}{ccccccccc}
0 & 0.2 & 0.1 & 0 & 0.5 & 0.3 & 0.1 & 0 & 0 \\
0.2 & 0 & 0.3 & 0 & 0.1 & 0 & 0.2 & 0.1 & 0 \\
0.1 & 0.3 & 0 & 0.4 & 0 & 0 & 0 & 0.6 & 0.1 \\
0 & 0 & 0.4 & 0 & 0.2 & 0 & 0.3 & 0.7 & 0.2 \\
0.5 & 0.1 & 0 & 0.2 & 0 & 0.7 & 0.1 & 0 & 0 \\
0.3 & 0 & 0 & 0 & 0.7 & 0 & 0.2 & 0.1 & 0.3 \\
0.1 & 0.2 & 0 & 0.3 & 0.1 & 0.2 & 0 & 0 & 0 \\
0 & 0.1 & 0.6 & 0.7 & 0 & 0.1 & 0 & 0 & 0.2 \\
0 & 0 & 0.1 & 0.2 & 0 & 0.3 & 0 & 0.2 & 0
\end{array}\right], \\
B & =\left[\begin{array}{cccccccccc}
0.1 & 0 & 0 & 0 & 0 & 0 & 0 & 0 & 0 \\
0 & 0.7 & 0 & 0 & 0 & 0 & 0 & 0 & 0 \\
0 & 0 & 0 & 0 & 0 & 0 & 0 & 0 & 0 \\
0 & 0 & 0 & 0 & 0.2 & 0 & 0 & 0 & 0 \\
0 & 0 & 0 & 0 & 0 & 0 & 0 & 0 & 0 \\
0 & 0 & 0 & 0 & 0 & 0.3 & 0 & 0 & 0 \\
0 & 0 & 0 & 0 & 0 & 0 & 0 & 0 & 0 \\
0 & 0 & 0 & 0 & 0 & 0 & 0 & 0 & 0 \\
0 & 0 & 0 & 0 & 0 & 0 & 0 & 0 & 0.1
\end{array}\right] .
\end{aligned}
$$


By computing, the eigenvalues of matrix $L+B$ are

respectively. Figures $1-5$ show the trajectories of multiagent systems with random initial states and $\alpha=0.2, h=0.4$, $\tau=0.3, \alpha=0.1, h=0.4, \tau=0.3, \alpha=0.1, h=0.4$, $\tau=0.2, \alpha=0.5, h=0.6, \tau=0.3$, and $\alpha=0.8$, $h=0.9, \tau=0.6$, respectively. Under Theorem 5, Figures 1 and 2 are the convergence situation, and Figure 3 is case of divergence. Under Theorem 6, Figures 4 and 5 are the results of simulations under the conditions of convergence and divergence.

\section{Conclusion}

In this paper, we have investigated the convergence problem of the fractional-order discrete-time multiagent system with a leader and sampling delay. We have obtained the convergence results depending on the sampling interval $h$, the fractional$\operatorname{order} \alpha$, and the sampling delay.

\section{Conflict of Interests}

The authors declare that there is no conflict of interests regarding the publication of this paper.

\section{Acknowledgments}

This work was supported in part by the National Natural Science Foundation of China under Grant no. 61473002, Beijing Natural Science Foundation Program no. 4132021, "The-Great-Wall-Scholar" Candidate Training-Plan of North China University of Technology, Construction Plan for Innovative Research Team of North China University of Technology, the Plan Training Project of Excellent Young Teacher of North China University of Technology, and the Special Project of North China University of Technology (no. XN085).

\section{References}

[1] W. Zhang, H. Su, X. Cai, and H. Guo, "A control lyapunov function approach to stabilization of affine nonlinear systems with bounded uncertain parameters," Circuits, Systems, and Signal Processing, vol. 34, no. 1, pp. 341-352, 2015.

[2] H. Su, G. Jia, and M. Z. Q. Chen, "Semi-global containment control of multi-agent systems with input saturation," IET Control Theory and Applications, vol. 8, no. 18, pp. 2229-2237, 2014.

[3] H.-Y. Yang, L. Guo, Y.-L. Zhang, and X.-M. Yao, "Movement consensus of complex fractional-order multi-agent systems," Acta Automatica Sinica, vol. 40, no. 3, pp. 489-496, 2014.

[4] H. Su, M. Z. Q. Chen, X. Wang, and J. Lam, "Semiglobal observer-based leader-following consensus with input saturation," IEEE Transactions on Industrial Electronics, vol. 61, no. 6, pp. 2842-2850, 2014.

[5] H. Su, M. Z. Q. Chen, J. Lam, and Z. Lin, "Semi-global leaderfollowing consensus of linear multi-agent systems with input saturation via low gain feedback," IEEE Transactions on Circuits and Systems I: Regular Papers, vol. 60, no. 7, pp. 1881-1889, 2013.

[6] H. Hu and Y. Zhu, "Controllability of impulsive fractional dynamical systems with distributed delays," Electronica Sinica, vol. 15, no. 4, pp. 34-37, 2012.

[7] H. Su and M. Z. Chen, "Multi-agent containment with input saturation on switching topologies," IET Control Theory and Applications, vol. 9, no. 3, pp. 399-409, 2015.

[8] B. Liu, H. Su, R. Li, D. Sun, and W. Hu, "Switching controllability of discrete-time multi-agent systems with multiple leaders and time-delays," Applied Mathematics and Computation, vol. 228, pp. 571-588, 2014.

[9] P. J. Torvik and R. L. Bagley, "On the appearance of the fractional derivative in the behavior of real material," Journal of Applied Mechanics, vol. 51, no. 2, pp. 294-298, 1984.

[10] C.-X. Zhu and Y. Zou, "Summary of research on fractionalorder control," Control and Decision, vol. 24, no. 2, pp. 161-169, 2009.

[11] W. Zhang, H. Su, and F. Zhu, "Full-order and reduced-order observer design for one-sided Lipschitz nonlinear systems with unknown inputs," Nonlinear Dynamics, vol. 79, no. 2, pp. 14691479, 2015.

[12] Y. Zhang, Study on Fractionl-Order Calculus and Its Applications, Northeastern University, Shenyang, China, 2008.

[13] Y. Cao, Y. Li, W. Ren, and Y. Chen, "Distributed coordination of networked fractional-order systems," IEEE Transactions on Systems, Man, and Cybernetics, Part B: Cybernetics, vol. 40, no. 2, pp. 362-370, 2010.

[14] Y. Cao and W. Ren, "Distributed formation control for fractional-order systems: dynamic interaction and absolute/relative damping," Systems and Control Letters, vol. 59, no. 3-4, pp. 233-240, 2010.

[15] Q. S. Zeng, D. Q. Feng, and G. Y. Cao, “The controllability and observability criteria of systems described by fractional differential equations," Journal of Zhengzhou University, vol. 25, no. 1, pp. 66-69, 2004.

[16] Z. Yanzhu and X. Dingyu, "Modeling and simulating transmission lines using fractional calculus," in Proceedings of the International Conference on Wireless Communications Networking and Mobile Computing (WiCom '07), pp. 3115-3118, IEEE, Shanghai, China, 2007.

[17] B. Liu, X. Han, J. Zhang, and D. Sun, "Convergence of fractionalorder discrete-time multi-agent systems with a leader," in Proceedings of the 34th Chinese Control Conference, Hangzhou, China, July 2015.

[18] F. Yu, N. Yu, and J. Wang, "Research on controllability for fractional order linear time-invariant systems," Journal of Chong University of Posts Telecommunications (Natural Science), vol. 19, no. 5, pp. 647-648, 2007.

[19] H.-Y. Yang, S.-W. Tian, and S.-Y. Zhang, "Consensus of multiagent systems with heterogeneous delays and leader-following," Acta Electronica Sinica, vol. 39, no. 4, pp. 872-876, 2011. 
[20] H.-Y. Yang, L. Guo, Y.-L. Zhang, and X.-M. Yao, "Delay consensus of fractional-order multi-agent systems with sampling delays," Acta Automatica Sinica, vol. 40, no. 9, pp. 2022-2028, 2014.

[21] K. Lin, "Analysis and comparision of different definition about fractional integrals and derivatives," Journal of MinJiang University, vol. 24, no. 5, 2003.

[22] K. Ogata, Discrete-Time Control System, Prentice-Hall, Englewood Cliffs, NJ, USA, 1995.

[23] Q. Li, N. Wang, and D. Yi, Numerical Analysis, Tsinghua University Press, 5th edition, 2008. 


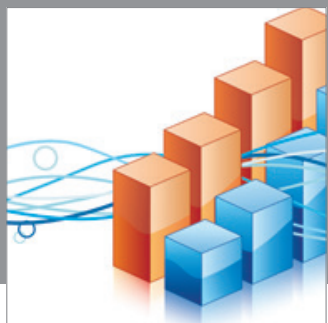

Advances in

Operations Research

mansans

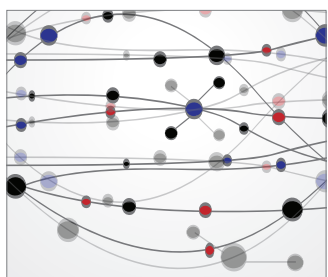

The Scientific World Journal
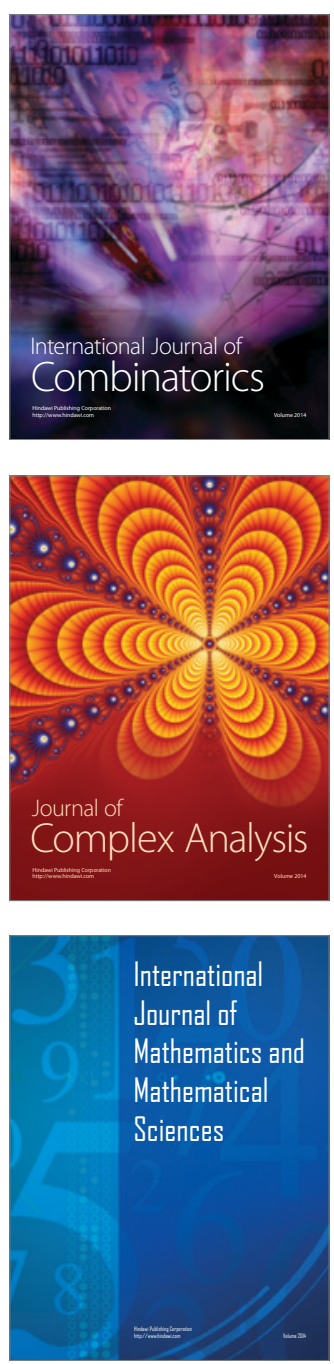
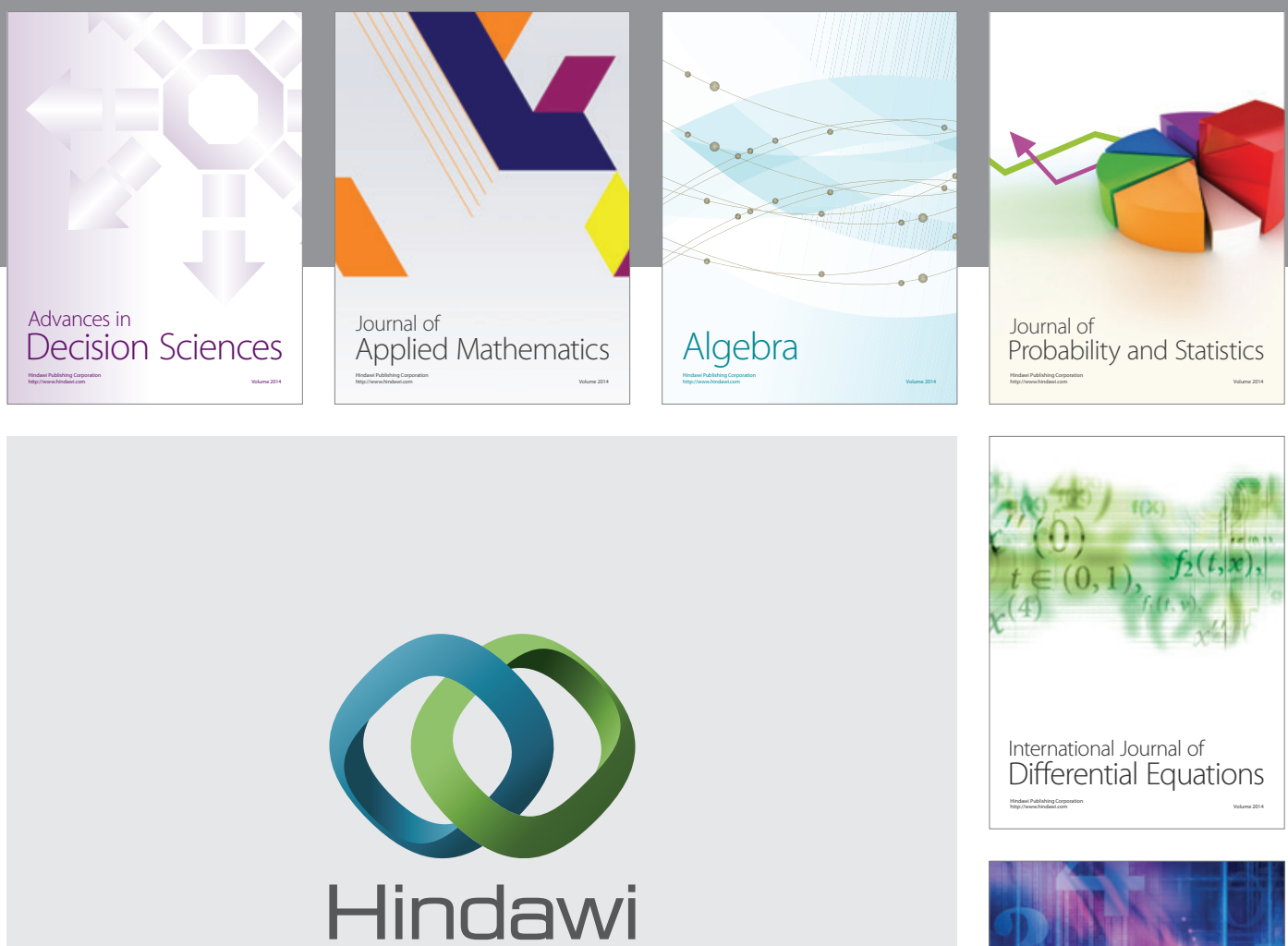

Submit your manuscripts at http://www.hindawi.com
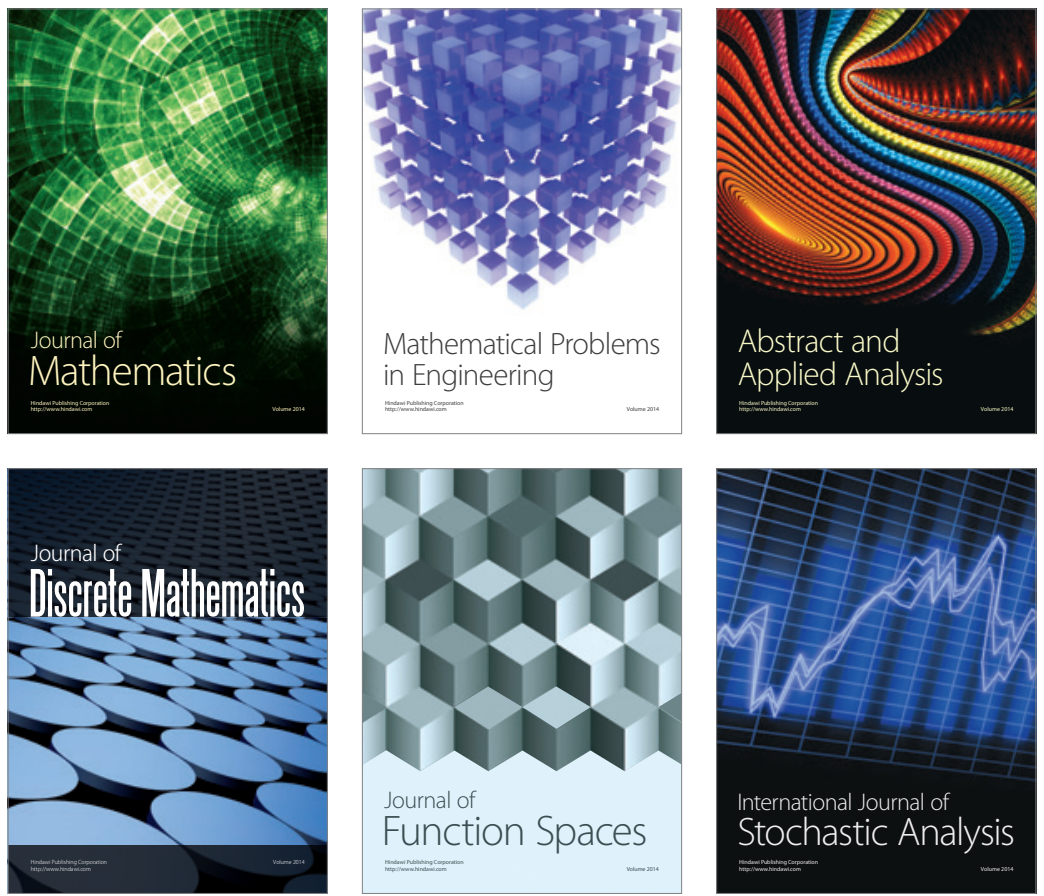

Journal of

Function Spaces

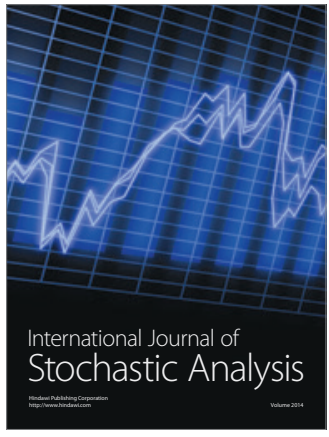

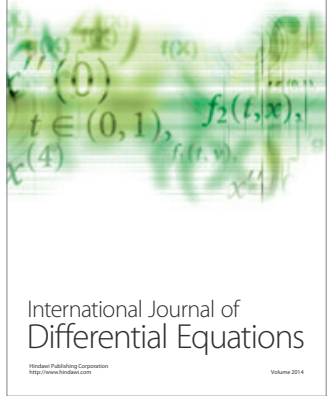
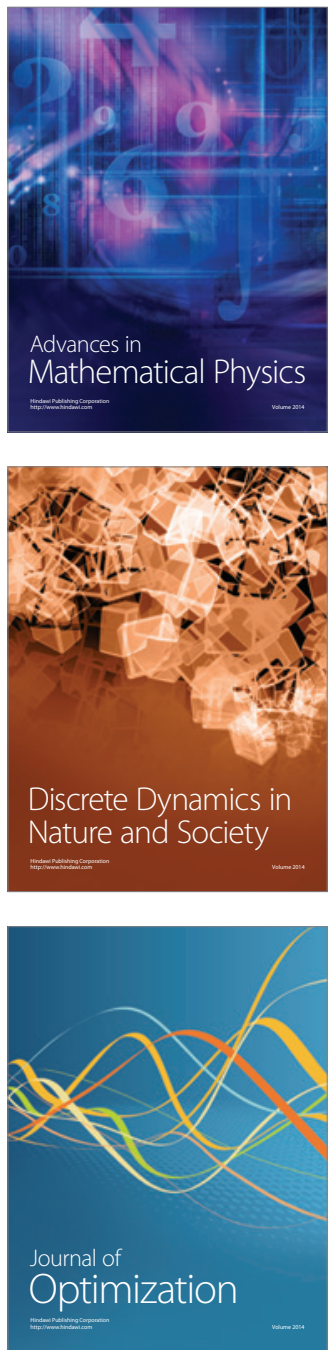